\title{
Is instant messaging going to replace SMS and e-mail as the medium of choice for direct customer communications?
}

Received: 1st October, 2003

\section{Shaun Doyle}

is CEO of Cognitive Box, a marketing technology solution provider. Before that he was VP Intelligent Marketing Solutions at SAS. In this role he worked with various parts of the SAS organisations to develop business-oriented solutions for marketing, in particular the SAS Marketing Automation (MA) Solution and SAS's industry-specific solutions for telco and retail banking. $\mathrm{He}$ was Founder and Chairman of Intrinsic, a campaign management vendor acquired by SAS in March 2001 . He is well known in the marketing industry and regularly participates in leading industry conferences. He is also author of a number of papers on the application of database marketing that have been published in the UK and elsewhere.

\begin{abstract}
This paper looks at instant messaging services and sees if there is a role for them as a direct marketing tool. It describes the key function provided by the three major service providers and describes potential scenarios for this type of functionality to be used to drive real-time customer communications.
\end{abstract}

\section{INTRODUCTION}

There is no doubt that the internet has changed the way people communicate. For many, e-mail has virtually replaced traditional letters and even telephone calls as the choice for correspondence. Every day, billions of e-mail messages are sent out. E-mail has been the most rapidly adopted form of communication ever known. In less than two decades, it has gone from obscurity to mainstream dominance.

In this fast-paced world, sometimes even the rapid response of e-mail is not fast enough. There is no way of knowing if the person the e-mail is being sent to is online at that particular moment or not. Also, if multiple e-mails are being sent back and forth with the same person, it is normally necessary to click through a few steps to read, reply and send the e-mail. This is one of the reasons why instant messaging (IM) has gained popularity.

Gartner ${ }^{1}$ predicts that the instant messaging will become the preferred communication method, exceeding e-mail traffic by 2006 for both business and consumer. The Radicati Group ${ }^{2}$ estimates that more than 45 per cent of all companies in the USA have employees who use instant messaging on a regular basis at work.

The author believes that the power of IM is far from being understood. Like short text messaging (SMS), its mobile equivalent, IM will have a significant impact on real-time marketing in the future.

This paper explains what IM is and how it works. It then explores how IM could be used to support marketing in the future. Its aim is to stimulate 


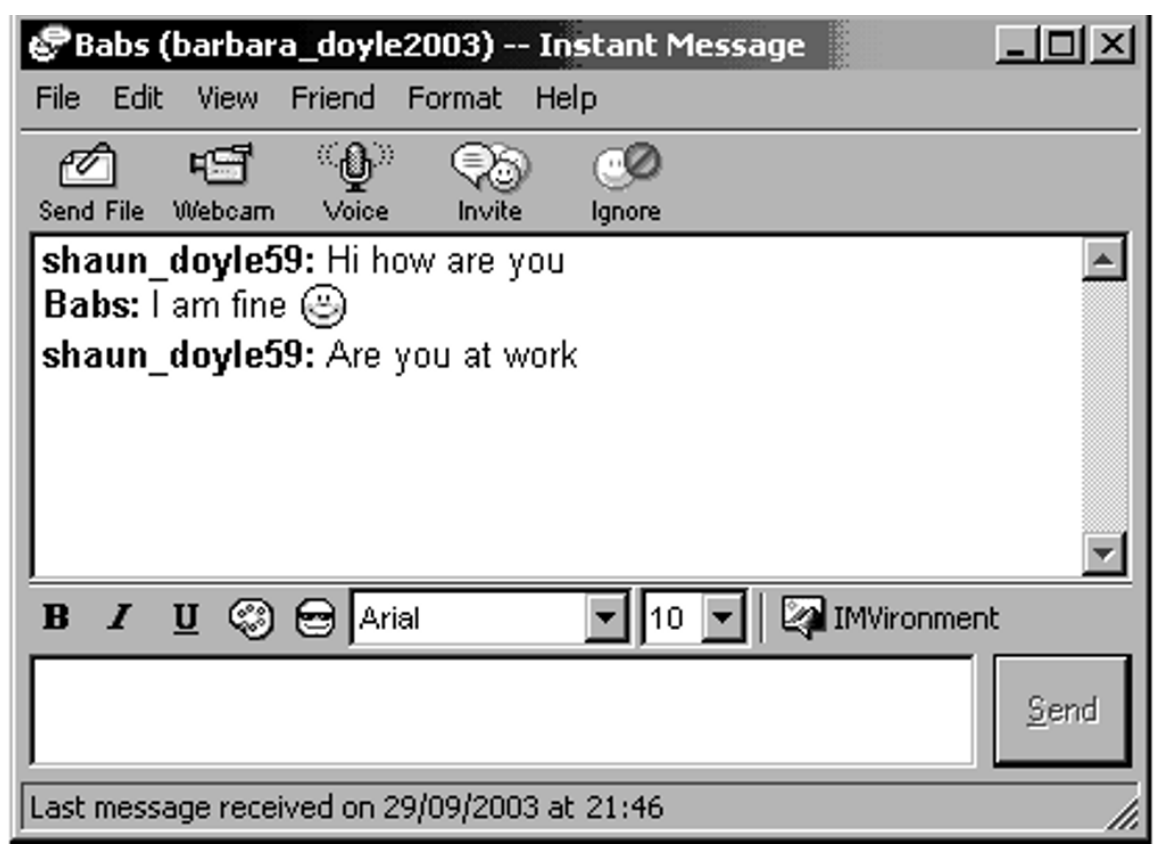

Figure 1: Sample dialogue box

discussion and open marketers' eyes to the potentially powerful tool that will make real-time communication with customers an economic possibility.

\section{INSTANT MESSAGING}

In its simplest form IM is an application that allows users to pass text messages to one another in real time across a network, most commonly the internet. IM allows a user to maintain a list of people with whom they wish to interact.

The user can send messages to any of the people on their list as long as that person is online. Sending a message opens up a small window where the user and peer can type in messages that both can see. But the IM solutions have evolved over the last few years to provide a wider range of functionality.

\section{STANDARD FUNCTIONALITY}

The following functionality is available from the major service providers.

\section{Enter user id and password}

The IM application allows the user to $\log$ onto the environment using a user-defined user name and password.

In many cases users create multiple user profiles, eg for home and personal use.

\section{Maintain status}

The IM application allows the user to maintain an availability status, which can be seen by other peers who are logged on. The following is a list of examples:

- available

- not available

— be right back

- busy

- not at home

- not in the office

- invisible mode (means the user will appear offline but can send messages)

— user-defined status.

This capability, called 'presence', allows 
other users to see that the individual is online and if they are available to have a dialogue. This is the key functionality of IM and is what differentiates it from e-mail and SMS.

\section{Maintain user profile}

If desired a user can create and share a profile. The following types of data are normally maintained:

$$
\begin{aligned}
& \text { - user name } \\
& \text { - real name } \\
& \text { - location } \\
& \text { - age } \\
& \text { - marital status } \\
& \text { - gender } \\
& \text { - occupation } \\
& \text { — e-mail address } \\
& \text { — interests. }
\end{aligned}
$$

In addition users can upload a personal photo. This profile information is normally searchable by other users.

\section{Manage account information}

The user can view and modify account information. At the moment most of these IM services are free, it will be interesting to see if this continues to be the case in the future.

\section{Maintain privacy settings}

The user can maintain the privacy level, setting instructions such as:

- ignore people who are not on my list

- ignore the following people

- show me as idle if I do not use my computer for XX minutes

- allow other users to see me

- allow other users to contact me.

This is one of the aspects of the communication method that empowers the user to control who can see and contact them.

\section{Send text message}

The user is able to:

- view a list of 'peers' who are online

- select a user and send a message

- ring a buzzer at the other user's end to attract attention.

A range of additional functions are available that allow the user to customise the communication process. These include:

Text formatting:

- font

- size

- colour

- bold

- underline

— italic

- colour effects.

Emoticons: These are icons that are used to reflect the user's emotions or feelings. They are embedded in the text message. The following are some samples:

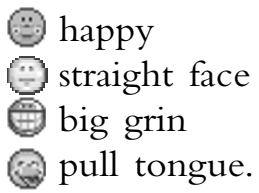

IMVironments: These are multimedia graphical components that can be used to personalise the user experience. The sample in Figure 2 is the most popular IMVironment on the Yahoo Messenger at the time of writing. These IMVironments often contain links to websites.

The following is a list of companies that have used IMVironments as a method to get customers to commercial websites: 


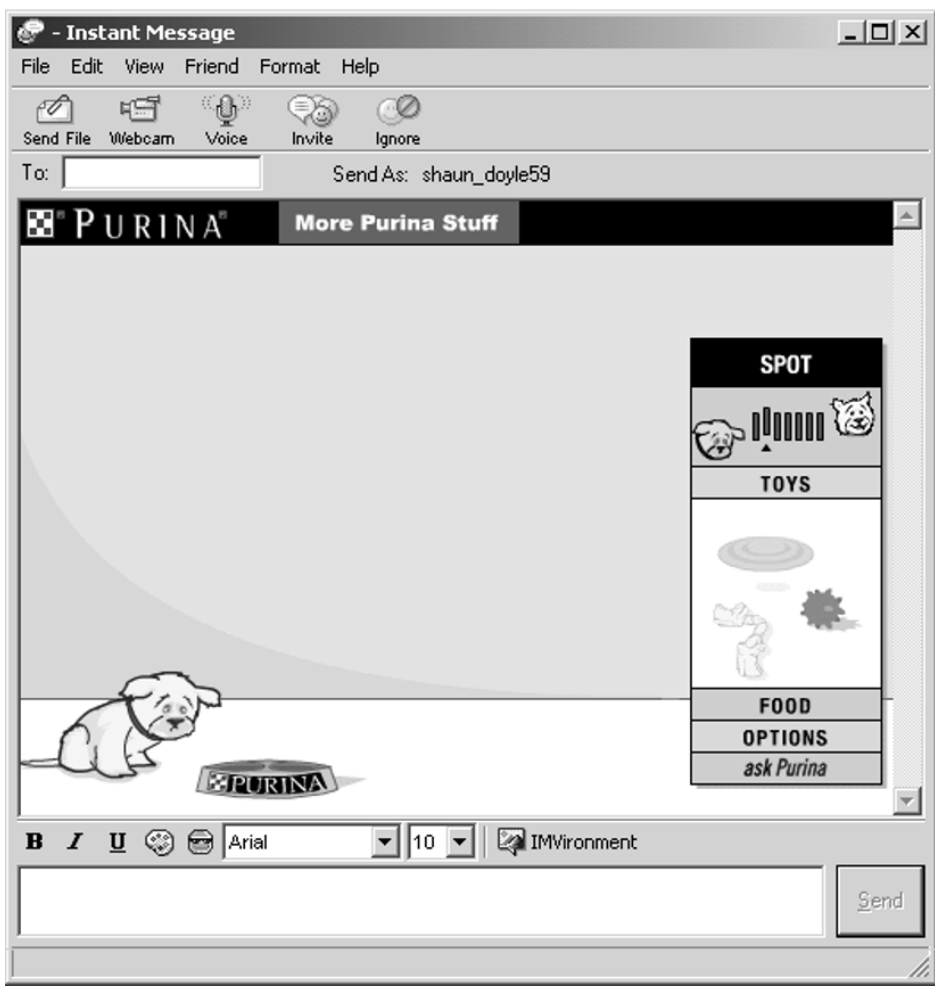

Figure 2: Sample IMVironment

- T-mobile

- Colgate

- Polo Jeans Inc

- Cingular

- Panasonic

- Mentos

- Purina.

In this case the suppliers are using the high volume of traffic on the IM service as source for customers.

\section{Send a file}

The user of the IM service can send a file to the other person involved in the dialogue. In some cases the file size can be much higher than those allowed by the e-mail system.

\section{Use voice}

The user is able to invite a peer to use voice communication (Figure 3).
The voice functionality can be used in conjunction with text messaging.

Although the quality is not as good as modern telephony the use of a good-quality headset improves the situation. As the performance of networks improve and the underlying technology evolves this quality issue will be addressed.

\section{Use webcam}

The user is able to invite a peer to use video in the form of webcam (Figure 4).

The video functionality can be used in conjunction with text messaging.

Although the quality is not perfect, high speeds and improved camera technology are leading to significant improvements.

\section{Invite to conference}

In the same ways as a user can engage in a dialogue with an individual, a user can 


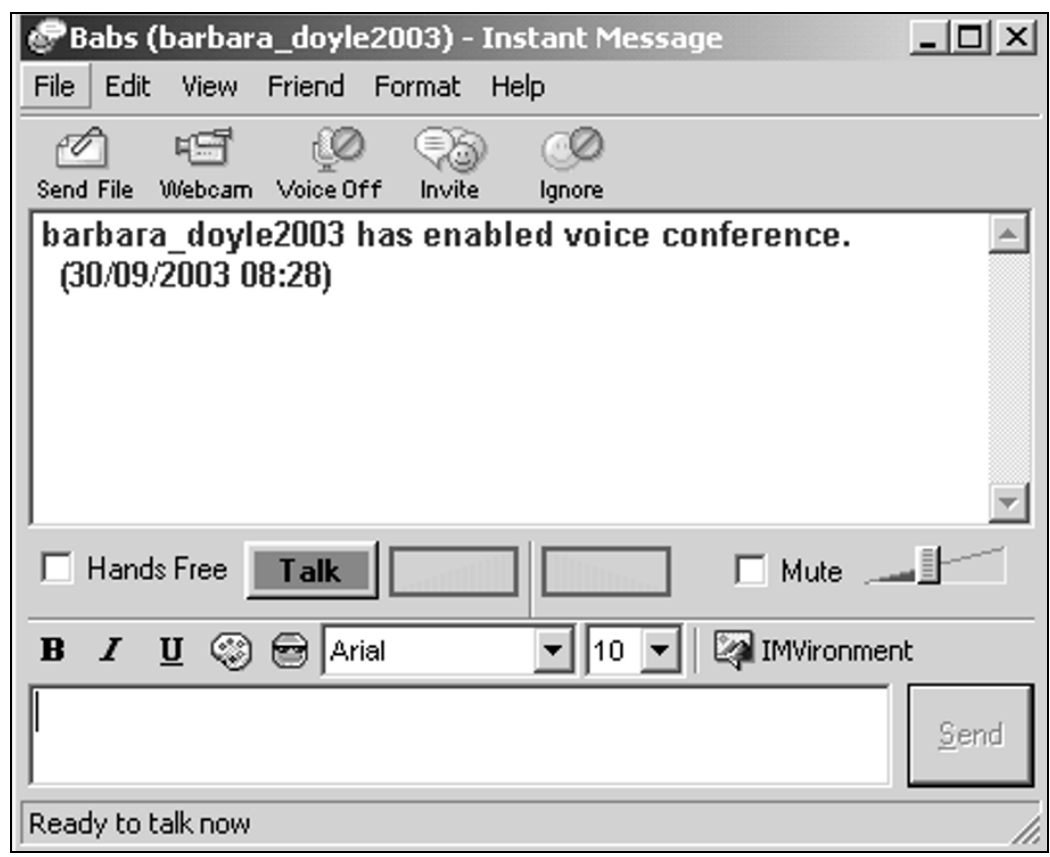

Figure 3: Sample dialogue box enabling voice conference

invite a number of other users to engage in a conference. These conferences can involve the use of voice, text and video or a combination.

\section{Enter chatrooms}

The user can enter a chatroom and engage in dialogue via text, voice or video. The chatroom works in the same way as a conference except the subject area is fixed.

Users can move between rooms, where various topics are discussed. They can create their own rooms to discuss a particular subject.

Rules have been set up for behaviour in the chatrooms, and in many cases are policed by the service providers.

\section{Start mobile session}

More recently, the key service providers have started providing the ability to execute text messaging to mobile devices such as mobile phones. The functionality is not quite as rich as over the PC (Figure 5).

The following telecom companies have integrated with one or more of the IM service providers:

- Cingular

- AT\&T Wireless

- Sprint

- Verizon Wireless

- T-mobile.

The main differences are in the pricing strategy of the organisation. SMS technology is used to carry the text message.

\section{Other}

In addition, the service providers are continuously trying to enhance their services, particularly as they start to apply the IM technologies in an enterprise context. The Radicati Group ${ }^{3}$ believe that the enterprise IM market will balloon during the next five years, to 


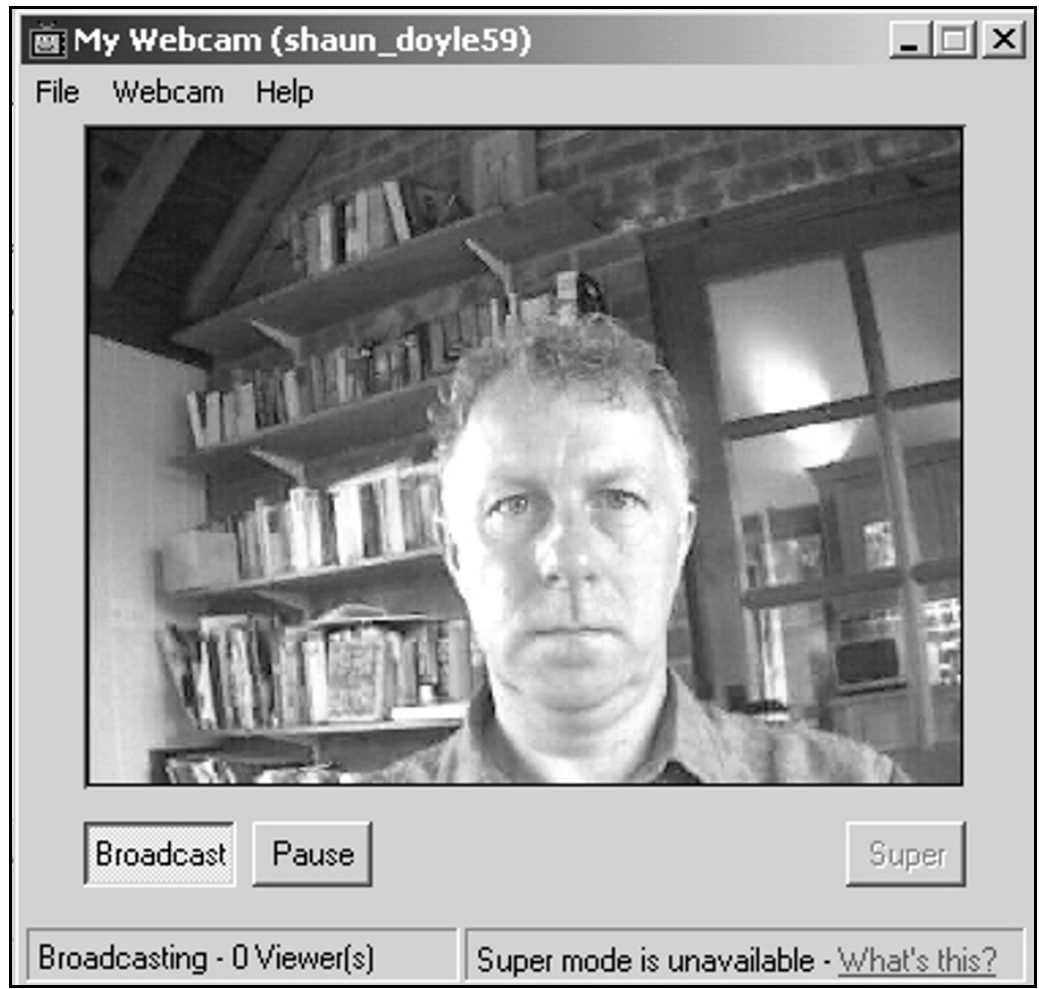

Figure 4: Sample voice cam screen

349 million users of IM in companies. That is a dramatic increase from the 60 million IM users in the workplace today.

Other areas of functionality include:

— higher levels of security

- ease of user administration

- access controls to public networks

— integrated logging

- integration with enterprise applications

— integration with enterprise e-mail systems

— streaming content.

\section{IM SERVICE PROVIDERS}

The primary suppliers of consumer-based instant messaging services are:

— Yahoo! Instant Messaging

- MSN Messenger

- AOL Instant Messenger

- ICQ Instant Messenger
There are several utilities, such as Odigo and Omni that combine various services. Odigo allows AIM, ICQ and Yahoo! Messenger contact lists to be combined. Omni lets the functionality of AIM, ICQ, MSN Messenger and Yahoo! Messenger, plus file sharing utilities, be combined all in one program.

\section{BUSINESS APPLICATIONS IN MARKETING}

The following are potential scenarios for the use of IM in a marketing context.

\section{Lead generation from IMVironments}

IMVironments are starting to be used for lead generation activity. The ability to create a multimedia experience within the context of the IM environment that provides a link to a corporate website is certainly generating increased web traffic. 


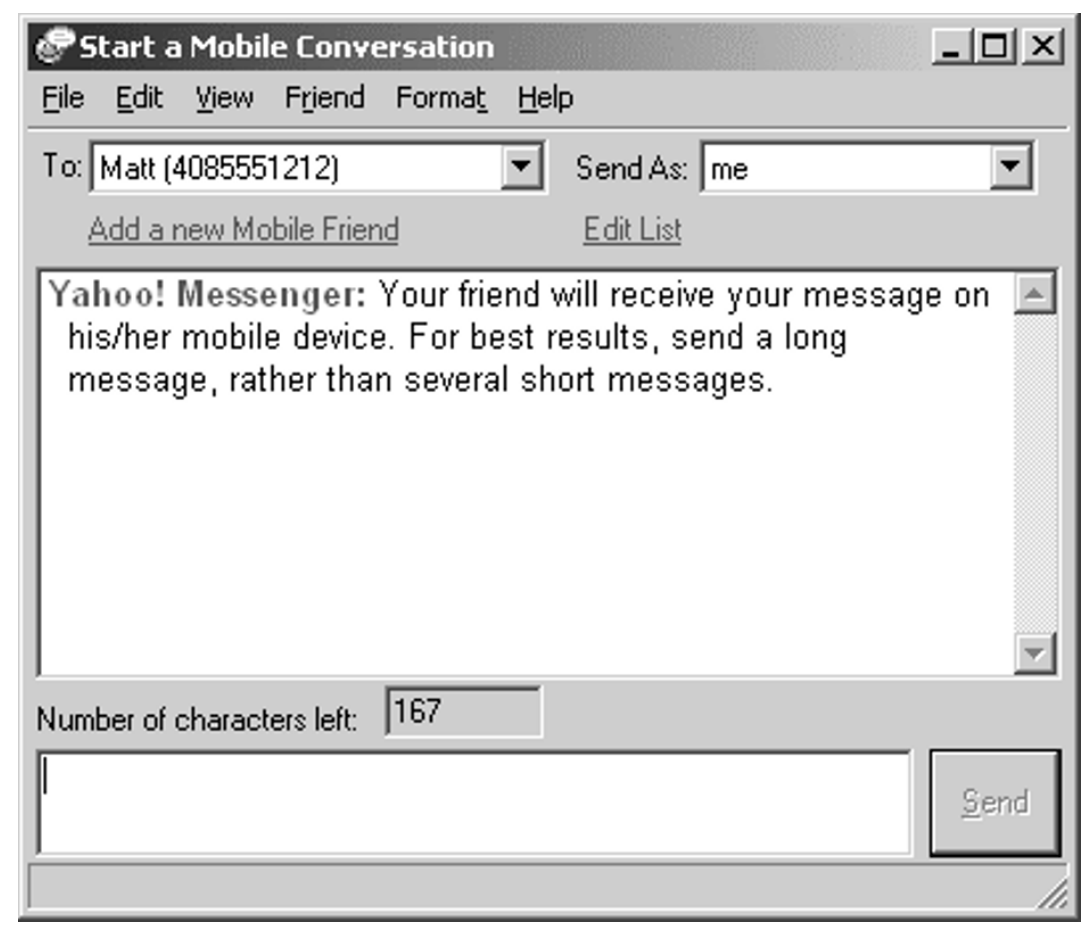

Figure 5: Sample dialogue box enabling mobile conversation

Yahoo! has more than 29 million users and that figure grew by 50 per cent over the previous year. They collectively send 17 billion messages a month or 550 million a day.

\section{Communicating using text messaging}

SMS has proved to be a valuable communication vehicle to individuals with mobile phones, but the high costs of sending and receiving SMS is stifling their business application in marketing. IM text messaging on the other hand is cheaper and allows a wider range of devices to send and receive the messages.

The ability to identify if a recipient is online and available also has significant advantages over SMS and e-mail.

\section{Communicating using voice}

The ability to switch between text and voice means that a customer can be initially engaged by text then migrated over to voice as part of the dialogue. Again the low-cost nature of the voice over IM is likely to result in the increased use of this type of telephone service.

There are quality issues at the moment but more widespread use by enterprises will drive improvements in quality.

\section{Multiparty conferencing}

The ability to allow other parties to join the dialogue means that small groups can participate in sales or marketing activities, eg discuss the benefits of a new product or service.

\section{Video}

The use of video to support the dialogue either in one-to-one or one-to-many situations enriches the quality of the interaction. The low-cost nature of the technology makes this a 
viable option for sales and marketing activities.

The use of web conferencing in the business-to-business sector has grown rapidly over the last few years; this type of event is likely to become more common in the business-to-consumer world.

The quality of web conferencing as part of IM may not be as good, but the lower costs will help to stimulate its usage.

\section{Sharing applications}

The ability to share applications such as PowerPoint in the context of a collaborative meeting opens up the possibility for sales presentations and other marketing activities.

\section{CASE STUDY}

Yahoo! and American Airlines (AA) are currently deploying an enterprise IM solution that will send an alert as a text message to customers when a flight is delayed. The customer is able to request an alternate flight using IM text messaging and if necessary engage an AA Staff member by voice using IM. The result is a low-cost real-time dialogue that results in improved customer service.

\section{CHALLENGES}

There are a number of challenges that will need to be addressed by the industry. These include:

- security

- protocol standards $\star$

— wireless mobility

— interoperability

- spam

- scalability

— integration.
* Standards are being addressed by the IETF $^{4}$ over the next few years.

\section{CONCLUSIONS}

The growth of IM as a real-time communication method is about to explode, fuelled by the enterprises' adoption of the technology and the increasing connectivity between the main service providers. Most of the technology market analysts predict that IM traffic will exceed that of e-mail in the next four to five years, IM becoming the preferred communication for both business and consumers. A few organisations have started to explore the potential role of IM as a direct communication vehicle. The initial results seem to be quite favourable. The control that the technology gives to the user means that some of the issues associated with e-mail and commercial SMS will be avoided. This is definitely an area that marketers should watch.

\section{Acknowledgments}

Websites:

Yahoo! Instant Messaging www.yahoo.com

MSN Messenger www.msn.com

AOL Instant Messenger www.aol.com

ICQ Instant Messenger www.iqc.com

(C) Shaun Doyle

\section{References}

1 Grey, M. C. (2003) 'IM key technology for real time enterprise', 7th February, Gartner Inc., www.gartner.com.

2 Plewes, A. (2003) 'How consumer IM vendors are targeting business users', The Radicati Group Inc., www.radicati.com.

3 Ibid.

4 IETF Secretariat, c/o Corporation for National Research Initiatives, 1895 Preston White Drive, Suite 100, Reston, VA 20191-5434, USA. Tel: +1 703620 8990; Fax: +1 7036209071. 